\title{
Periostin, a matrix specific protein, is associated with proliferation and invasion of pancreatic cancer
}

\author{
QI-WEN BEN ${ }^{1}$, XIAO-LONG JIN ${ }^{2}$, JUN LIU $^{1}$, XIA CAI $^{3}$, FEI YUAN ${ }^{2}$ and YAO-ZONG YUAN ${ }^{1}$
}

\begin{abstract}
Departments of ${ }^{1}$ Gastroenterology and ${ }^{2}$ Pathology, Ruijin Hospital, Shanghai Jiaotong University, School of Medicine;
${ }^{3}$ Center Laboratory, Renji Hospital, Shanghai Jiaotong University, School of Medicine, Shanghai, P.R. China
\end{abstract}

Received October 22, 2010; Acceped December 15, 2010

DOI: $10.3892 /$ or.2011.1140

\begin{abstract}
Overexpression of periostin is present in various malignant tumors and correlates with disease progression. However, its clinicopathological significance in pancreatic cancer is currently not known. Expression of periostin was analyzed by RT-PCR and Western blotting in pancreatic cancers and cell lines. Using immunohistochemistry, expression of periostin in pancreatic cancers was evaluated according to factors influencing overall survival with Kaplan-Meier analysis. Ectopic expression of periostin was used to examine the effects of periostin on proliferation and invasiveness of pancreatic cancer cells in vitro. There was no detectable periostin mRNA and protein expression in the 4 pancreatic cell lines. Expression of periostin was found to be up-regulated in pancreatic cancer compared to the adjacent tumor free (TF) tissues by Western blotting. The positive ratio of periostin expression in the neoplastic stroma was significantly correlated with the depth of invasion $(\mathrm{p}=0.007)$ and lymph node metastasis $(p=0.027)$. Survival analysis showed that stromal or epithelium expression of periostin was associated with poor survival ( $\mathrm{p}=0.035, \mathrm{p}=0.022$, log-rank test, respectively). In vitro studies showed that periostin was able to promote proliferation and invasiveness of pancreatic cancer cells. These results suggest that periostin may be involved in the progression and invasion of pancreatic cancer.
\end{abstract}

\section{Introduction}

Pancreatic cancer is one of the most malignant gastrointestinal tumors, accounting for over 30,000 deaths yearly in the United States alone (1). In China, pancreatic cancer is the sixth leading cause of malignancy-related death, with an overall cumulative 5-year survival rate of $1-3 \%$

Correspondence to: Dr Yao-Zong Yuan, Department of Gastroenterology, Ruijin Hospital, Shanghai Jiaotong University, 197 Ruijin Er Road, Shanghai 200025, P.R. China

E-mail: yaozong_yuan28@yahoo.com.cn

Key words: immunohistochemistry, pancreatic cancer, periostin, prognosis, invasion, migration
(2). At the time of diagnosis, only $10-15 \%$ of these cancers are typically found to be resectable (1), due to the presence of locally advanced disease or distant metastases. Effective systemic therapy for advanced or recurrent disease is currently unavailable (3). Therefore, research efforts have been increasingly focused on elucidation of molecular targets and on development of novel therapeutic approaches for this disease.

Periostin (PN), originally designated osteoblast-specific factor 2 , has a typical signal peptide sequence at its $\mathrm{N}$ terminus and four repeated domains. Each of the latter shares a structural homology to insect fasciclin I $(4,5)$. The human periostin is found on chromosome $13 q$ (6), which encodes a protein of 811 amino acids with a molecular weight of $90.2 \mathrm{kDa}(4)$. Periostin is known to play a role in bone and tooth formation and maintenance of structure in these tissues (5). Periostin-null mice exhibit incisor enamel defects, dwarfism (7) and impaired scar formation in the infarct zone after acute myocardial infarction (8).

The expression of periostin is reported to be associated with both tumor suppression and tumor progression in a number of human cancers, and its role in tumorigenesis remains controversial. In human tumors, including head and neck cancer (9), breast cancer $(10,11)$, pancreatic cancer $(12,13)$, non-small cell lung cancer (NSCLC) (14) and colon cancer (15), periostin expression has been shown to be upregulated; however, this is not true for bladder cancer (16). Re-expression of periostin in colon cancer cells or in head and neck cancer cells promoted tumorigenicity in nude mice $(9,15)$. In addition, Erkan et al demonstrated that periostin was produced solely by pancreatic stellate cells (PSCs), which created a tumor-supportive microenvironment (12). Specifically, periostin is suggested to promote the invasiveness or growth rate and confer resistance to hypoxia in pancreatic cancer cells via the $\alpha 6 \beta 4$ integrin complex (17). Accordingly, periostin is reported to be a ligand for $\alpha \mathrm{v} B 3$ and $\alpha \mathrm{v} 35$ integrins inducing integrin-dependent cell adhesion and motility (18).

In this study, we relied on semi-quantitative immunohistochemistry to investigate periostin expression in pancreatic cancer and to correlate it with clinicopathological features of this tumor. Next, we also examined the role of periostin in pancreatic cancer growth and progression in vitro. Based on these studies, we verified that periostin has a critical role in pancreatic cancer progression. 


\section{Materials and methods}

Study population, follow-up and TMA construction. The study was approved by the ethics committee of the chamber of physicians of Ruijin Hospital, Shanghai, China. The cohort of study population and tissue microarray (TMA) construction were as described previously (19). Briefly, 94 patients who underwent potentially curative surgery for pancreatic cancer were recruited for this study between January 1, 2002 and December 31, 2007. All cases were staged according to the guidelines of the International Union Against Cancer (2002) (20). Patient follow-up was obtained through reviewing the hospital records, contacting with family members of the patients, or the Cancer Registry of Shanghai until November 1, 2008, with a median observation time of 20 months (range from 3 to 45 months). Original paraffin-embedded specimens were used to construct a TMA. Duplicates of 1-mm diameter cylinders from tumor center for each case and 1 1.0-mm tissue core from 'normal' adjacent pancreas for 22 patients were punched using an automated tissue arrayer (Beecher Instruments, Sun Prarie, Wisconsin). Thus, a tissue microarray block containing 210 cylinders was constructed.

Immunohistochemistry and scoring. The TMA slides were analyzed by immunostaining using the Dako Envision system (Dako, Carpinteria, CA) as described previously. Following quenching of endogenous peroxidase activity and blocking of non-specific binding, the slides were incubated with polyclonal antibody to periostin (Abcam, Cambridge, UK) in 1:200. Assessment for the abundance of periostin expressing cells was performed by two experienced pathologists (J.X. and Y.F.). Neoplastic epithelial cell staining was considered positive if the chromogen was detected in at least $10 \%$ of the cells within a microscopic field. Staining intensity included 4 scores: no staining (score 0), weak staining (score 1), moderately positive (score 2), and strongly positive (score 3 ). Scores 0 and 1 were regarded as negative and scores 2 and 3 as positive. Focal, multifocal, or diffused stainings were also scored. For neoplastic stroma, the immunohistochemical staining of anti-periostin were evaluated and classified by the area of positive-staining stroma into negative $(<25 \%)$ and positive $(>25 \%)$.

Cell lines and tissue sample. The four established pancreatic cancer cell lines (AsPC-1, SW-1990, BxPC-3 and Panc-1) in this study were obtained from the American Type Culture Collection (ATCC, Manassas, VA). Cells were grown in RMPI-1640 medium (Invitrogen, Carlsbad, CA) supplemented with $10 \%$ heat-inactivated fetal bovine serum (FBS) and 100 units/ml of penicillin-streptomycin (Invitrogen) under the following conditions: $5 \% \mathrm{CO}_{2}$ in air at $37^{\circ} \mathrm{C}$.

$R T$-PCR. RT-PCR were performed as previously described (21). Briefly, after cDNA template was synthesized according to the manufacturer's protocol, PCR was performed on $2 \mu \mathrm{l}$ of RT products in a $25-\mu 1$ reaction mixture. An internal control gene, $\beta$-actin, was included to control for the amount of RNA. The amplification products were then electrophoresed on $1.5 \%$ agarose gels.
Western blotting. Protein was isolated from exponentially growing cells and fresh-frozen pancreatic tissue samples. After protein concentrations measured, the proteins were transferred to polyvinylidene fluoride membranes and blotted with human monoclonal anti-periostin (R\&D Systems, Minneapolis, MN). Immunoreactive bands were detected by using chemiluminescence (Pierce Chemical Co., Rockford, IL) according to the manufacturer's instructions.

Construction of recombinant adenoviruses and cell transfection. Periostin full-length cDNA was cloned into a pDC316 carrier plasmid (Benyuanzhengyang, Beijing, China) to generate pDC316-PN. The plasmid pDC316-PN and the skeleton plasmid pBHG-fiber5/35 (Benyuanzhengyang) were co-transfected into HEK293 cells, and which yielded the recombinant Ad5/F35-PN plasmid. Successful recombination was confirmed by observation of cytotoxicity as well as by PCR. The control vector Ad5/F35-Null was obtained by the same methods. Cell infections were done by using 0-100 multiplicities of infection (MOI) per target cell. The recombinant adenovirus Ad5-PN was used after purification, characterization, and titration of the viral infectivity by plaque assay.

Proliferation assay. Proliferation of cells infected with Ad5PN or Ad5-null at MOI of $100 \mathrm{pfu} /$ cell was determined by MTT assay at $12,24,48$, and $72 \mathrm{~h}$. The mock reaction contained no virus. Absorption was measured at $490 \mathrm{~nm}$. The experiment was repeated three times.

Soft agar colony formation assay. Assays of colony formation in soft agar were done in 6-well plates with a base layer containing $0.6 \%$ agar. Cells $\left(1 \times 10^{4}\right)$ were plated onto the previously prepared underlayers $(1.2 \%$ agar $)$. The plates were incubated at $37^{\circ} \mathrm{C}$ in a humidified $5 \% \mathrm{CO}_{2}$ atmosphere for 10 days, and afterwards, colonies $>50 \mu \mathrm{m}$ were counted 15 days after plating.

Cell migration and invasion assay. Cell migration and invasion assays were conducted by using $8-\mu \mathrm{m}$ pore size transwell chambers (Chemicon). After cells $\left(1.5 \times 10^{6}\right.$ cells $\left./ \mathrm{ml}\right)$ were placed in the upper compartment, the lower chambers of the transwell were filled with $500 \mu 1$ of medium with $10 \% \mathrm{FBS}$ as a chemoattractant. The plates were incubated at $37^{\circ} \mathrm{C}$ for 24 and $48 \mathrm{~h}$. Non-invasive cells and matrigel were removed by scraping with sterile cotton swabs. After stained with hematoxylin, the migrated or invaded cells were counted under a microscope at x400 magnification. Assays were done thrice, and 10 fields were randomly selected and counted for each assay.

Statistical methods. All statistical analyses were performed with SPSS13.0 software (SPSS, Inc., Chicago, IL). To compare the mean difference in various clinicopathological factors between groups, the $\chi^{2}$ test and Fisher's exact test were used. Survival curves were calculated according to the method of Kaplan and Meier and p-values were evaluated by the log-rank test. The difference between means was performed with Student's t-test. For all analyses, a two-sided p-value of $<0.05$ was defined as statistically significant. 

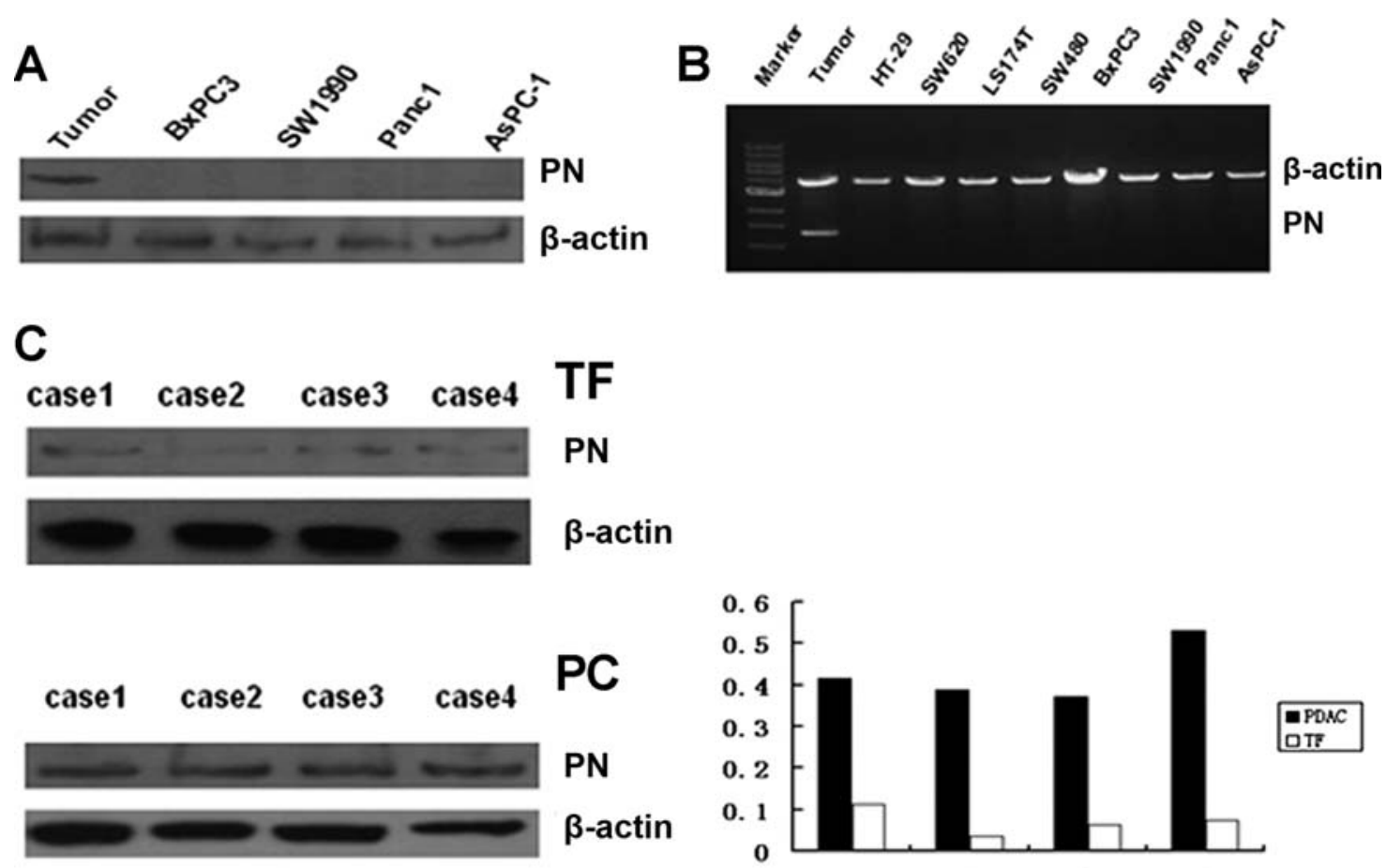

Figure 1. Periostin expression in a panel of pancreatic cancer cell lines and tissues. (A) Protein expression of periostin in cell lines assayed by Western blot analysis with a monoclonal anti-periostin antibody. Human periostin protein was not present in pancreatic cancer cell lines. (B) RT-PCR was used to assay periostin mRNA in cell lines. Human periostin mRNA was not present in pancreatic cancer cell lines and colon cancer cell lines (previously reported). (C) Periostin was differentially expressed in pancreatic cancer samples compared with adjacent tumor free (TF) pancreas from the same patient. The expression of B-actin was used as an internal control. Bands were quantified by Quantity One software (Bio-Rad). M, Marker; T, human colorectal cancer tissue; PN, periostin; TF, tumor-free tissues; PDAC, pancreatic ductal adenocarcinoma.

\section{Results}

Expression of periostin in human pancreatic cancer cell lines and tissues. As shown in Fig. 1A and B, periostin mRNA and protein were not present in the four cell lines (AsPC-1, SW-1990, BxPC-3, Panc-1) after RT-PCR and Western blot analysis. In order to determine the expression of periostin protein in human pancreatic cancer tissues, we performed Western blot analysis on four tumors and matched tumor-free tissues. The average periostin expression level (periostin/ß3actin signal intensity ratio) was significantly higher in tumors than in matched TF tissues ( $\mathrm{p}<0.05$, Fig. 1C).

Expression of periostin on TMA. To determine the distribution and clinical significance of periostin in pancreatic cancer, we analyzed the expression pattern of periostin on TMA by using immunohistochemistry. In adjacent normal pancreas, periostin was slightly expressed in capillary endothelial cells, fibroblasts; no or faint expression was found in ductal cells (Fig. 2A). In pancreatic cancer, strong periostin expression was observed close to the neoplastic cells in the neoplastic stroma (Fig. 2B and C). Interestingly, most of the neoplastic epithelium, only moderate levels of periostin expression could be observed (Fig. 2D and E). Collectively, up to $80 \%$ of tumors were strongly positive for periostin staining in the neoplastic stroma, whereas about $30 \%$ showed strong staining in neoplastic epithelium.

We then examined whether periostin expression is correlated with the development of pancreatic cancer. Periostin expression was significantly up-regulated in pancreatic cancer than that of matched 'normal' tissues $(\mathrm{p}<0.001$, data not shown). In addition, the positive expression of periostin in the neoplastic stroma was significantly correlated with depth of invasion $(\mathrm{p}=0.007)$ and lymph node metastasis $(\mathrm{p}=0.027)$ (Table I). Survival analyses showed that the median survival time in periostin negative group (19.4 months) was significantly longer than that in periostin positive group (8.7 months, $\mathrm{p}=0.035$, Fig. 3A). Interestingly, elevated expression of periostin in neoplastic epithelium was also associated with poor prognosis in pancreatic cancer ( $\mathrm{p}=0.022$, log-rank test), although which was not correlated with the advanced clinicopathological factors in this disease (Table I and Fig. 3B).

Periostin overexpression promotes anchorage-independent growth. To determine the effect of periostin on pancreatic cancer development, we constructed periostin recombinant adenovirus plasmid Ad5-PN. After the recombinant adenovirus vectors were constructed successfully, the virus was generated at $1-5 \times 10^{9} \mathrm{pfu} / \mathrm{ml}$ in titer. Human pancreatic cancer cells BxPC-3 or Panc-1, which had no expression of periostin, were infected with Ad5-PN adenovirus vectors. Our data showed that infection of BxPC-3 cells with Ad5-null at a MOI of $100 \mathrm{pfu} /$ cell conferred transgene expression in nearly $97 \%$ of cells by detecing green fluorescent protein in microscope (Fig. 4A).

As shown in Fig. 4B, after virus infection, periostin did not significantly promote cell growth compared with parental cells or mock infection. However, the effect of periostin 

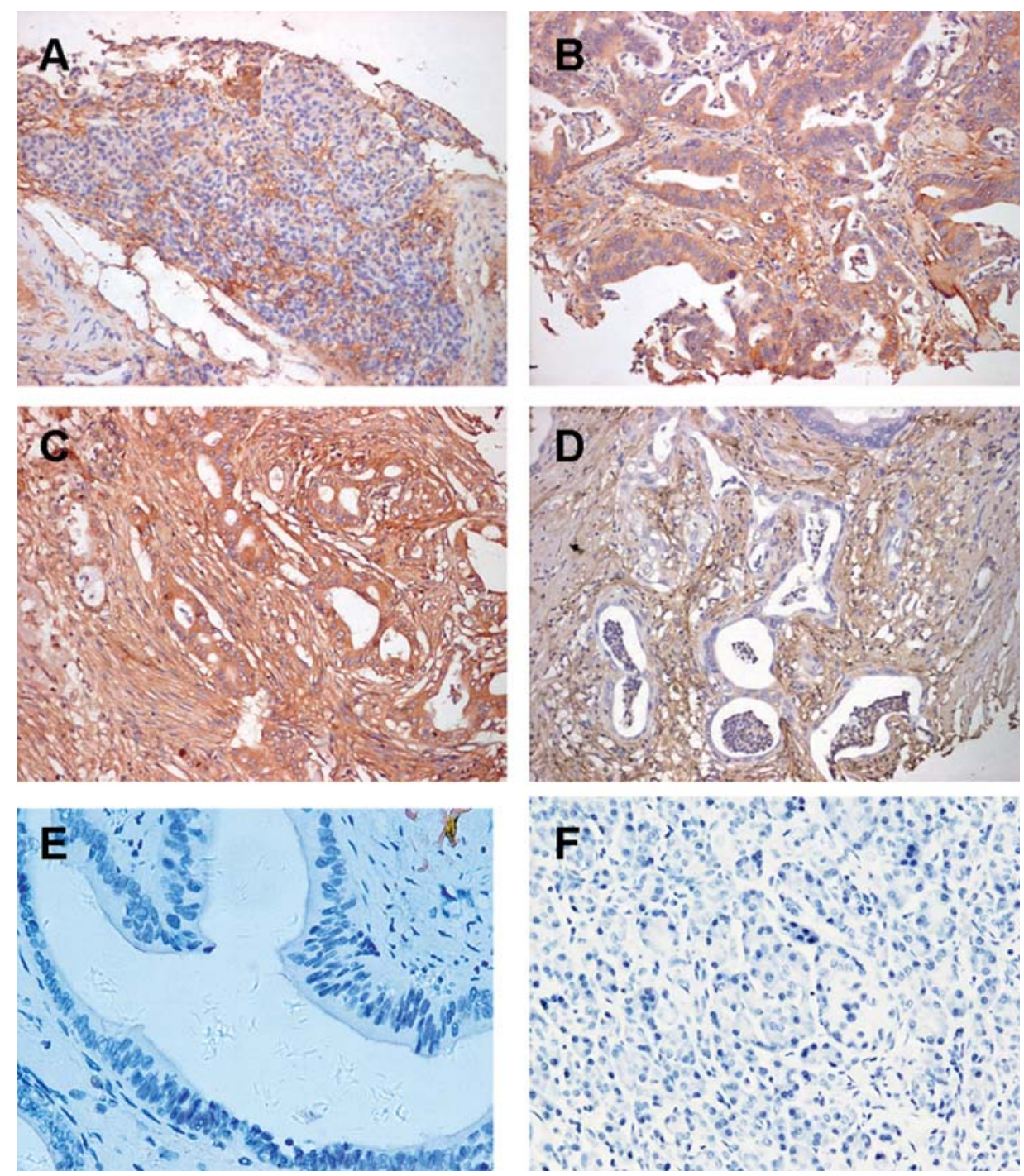

Figure 2. Immunohistochemistrical staining of periostin in human pancreatic tissues. The tissue cores were immunostained with a polyclonal periostin antibody. (A) Faint stromal immunostaining in adjacent 'normal'tissues. (B) Moderate staining in neoplastic stroma and strong staining in epithelium. (C) Strong periostin staining in neoplastic stromal and epithelial cells. (D) Strong staining in neoplastic stroma and no staining in neoplastic epithelium. (E) No staining in neoplastic epithelium. (F) Negative controls. (A-D, original magnification x200; E and F, magnification x400).

was more pronounced on promoting anchorage-independent growth, measured by the number of colony formation in soft agar assays. This finding was also found for another cell line, Panc-1, which showed more numerous and larger colonies with a soft agar assay (Fig. 4C).

Periostin promoted cell motility and invasion in vitro. To examine whether periostin had a role in cancer cell metastatic processes, we performed cell migration and invasion assays using transwell chambers, respectively. Surprisingly, periostin infected cells migrated faster at 24 and $48 \mathrm{~h}$ than did controls (Fig. 5A and B). With the invasion assay, the number of invaded periostin-overexpression cells was significantly more than the number of invaded parental cells or GFP infected cells (Fig. 5C and D).

\section{Discussion}

To develop adequate therapeutic modalities against pancreatic cancer, an increased number of molecular markers have been identified to correlate with tumor development and progression in this disease (22-24). The present study indicated an overall higher expression of periostin in pancreatic cancer, especially in the tumor-associated stroma. We also found a correlation between periostin expression in neoplastic stroma and epithelium and overall survival of patients with pancreatic cancer after potentially curative surgery. In addition, endogenous periostin might efficiently promote invasion and anchorageindependent growth of pancreatic cancer cells, which highlights its potential role as a therapeutic target.

The source of periostin in tumor is still under debate. Recent researches indicated that periostin protein is a matrixspecific protein, with high expression in the stromal cells surrounding the carcinoma epithelium $(12,25-28)$, whereas other experiments suggested that periostin was detected in cancer cells $(17,29)$. Interestingly, our previous study on colorectal cancers found that serum periostin levels after curative surgery showed a significant decrease below preoperative levels (21). In the present study, we found that periostin mRNA and protein were not present in pancreatic 
Table I. Clinicopathological variables with the epithelial or stromal expression of periostin in pancreatic cancers.

\begin{tabular}{|c|c|c|c|c|c|c|c|}
\hline \multirow[b]{2}{*}{ Parameters } & \multirow[b]{2}{*}{$\mathrm{n}$} & \multicolumn{3}{|c|}{ Periostin epithelial expression } & \multicolumn{3}{|c|}{ Periostin stromal expression } \\
\hline & & Negative & Positive & P-value ${ }^{a}$ & Negative $(<25 \%)$ & Positive (>25\%) & P-value ${ }^{a}$ \\
\hline Age (years) & & & & 0.382 & & & 0.419 \\
\hline$\leq 60$ & 54 & 36 & 18 & & 8 & 46 & \\
\hline$>60$ & 40 & 30 & 10 & & 9 & 31 & \\
\hline Gender & & & & 0.527 & & & 0.173 \\
\hline Male & 55 & 40 & 15 & & 7 & 48 & \\
\hline Female & 39 & 26 & 13 & & 10 & 29 & \\
\hline Tumor classification & & & & 0.135 & & & 0.007 \\
\hline $\mathrm{T} 1$ & 8 & 5 & 3 & & 4 & 4 & \\
\hline $\mathrm{T} 2$ & 25 & 14 & 11 & & 7 & 18 & \\
\hline $\mathrm{T} 3$ & 61 & 47 & 14 & & 6 & 55 & \\
\hline Node involvement & & & & 0.134 & & & 0.027 \\
\hline No & 61 & 46 & 15 & & 15 & 46 & \\
\hline Yes & 33 & 20 & 13 & & 2 & 31 & \\
\hline Histological grading & & & & 0.460 & & & 0.133 \\
\hline $1 / 2$ & 69 & 47 & 22 & & 8 & 61 & \\
\hline $3 / 4$ & 25 & 19 & 6 & & 9 & 16 & \\
\hline Tumor size (cm) & & & & 0.590 & & & 0.287 \\
\hline$<4$ & 43 & 29 & 14 & & 10 & 33 & \\
\hline$\geq 4$ & 51 & 37 & 14 & & 7 & 44 & \\
\hline Vascular invasion & & & & 0.544 & & & 0.538 \\
\hline Yes & 56 & 38 & 18 & & 9 & 47 & \\
\hline No & 38 & 28 & 10 & & 8 & 30 & \\
\hline
\end{tabular}

aTested by the $\chi^{2}$ test and Fisher's exact test.
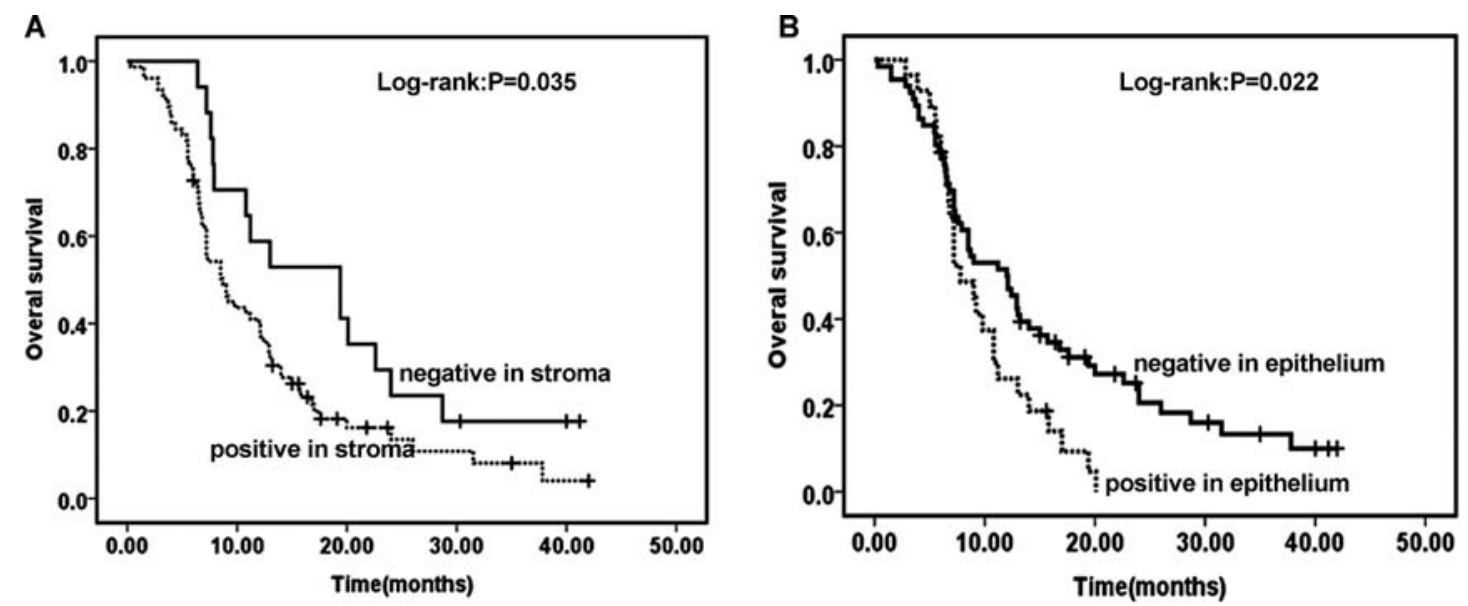

Figure 3. Kaplan-Meier analysis of overall survival of patients with pancreatic cancer according to periostin expression. The median survival time in the stromal (A) or epithelial (B) expression-negative group were significantly longer than those in the stromal or epithelial expression-positive group $(\mathrm{P}=0.035$, $\mathrm{P}=0.022$, log-rank test, respectively).

cancer cell lines, and that no or faint periostin protein was present in adjacent human non-cancerous pancreas, while expression of periostin was up-regulated in pancreatic cancer tissue samples. In addition, we also found that up to $80 \%$ of 
A

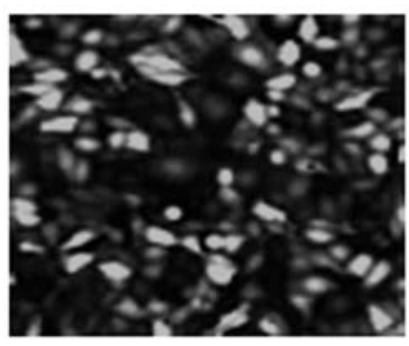

\section{00490}

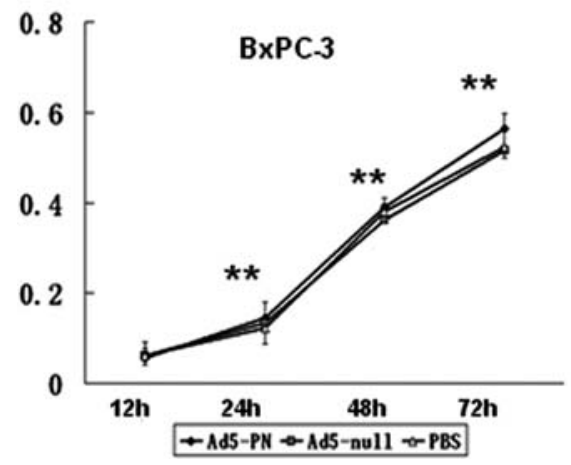

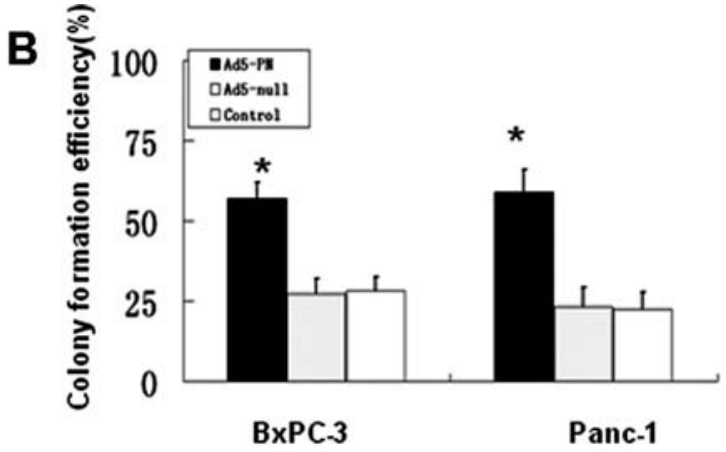

D 00490

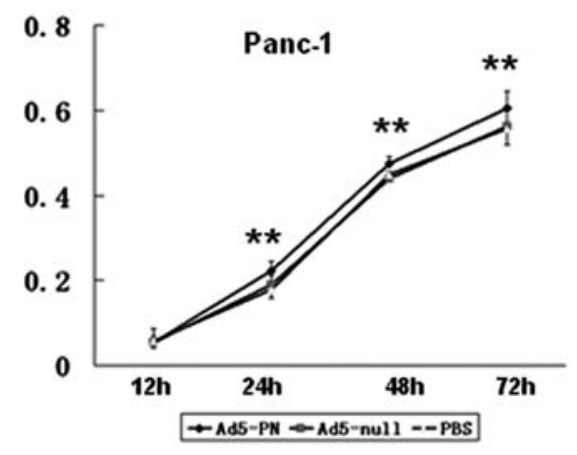

Figure 4. Cell infection of recombinant adenovirus and effects of periostin on cell proliferation and colony formation in vitro. (A) BxPC-3 cells infected with Ad5-null at a multiplicity of infection (MOI) of $100 \mathrm{pfu} / \mathrm{cell}$ conferred transgene expression in nearly $97 \%$ of cells by detecting fluorescent protein in microscope (x200). (B) BxPC-3 or Panc-1 cells were infected with Ad5-PN or Ad5-null at MOI of 100. Cells were also treated by phosphate-buffered saline (PBS) as a mock infection. The viability of cells was determined by MTT (3,-[4,5-dimethylthiazol-2-yl]-2,5-diphenyltetrazolium bromide) assay at 12, 24, 48, and $72 \mathrm{~h}$. Cell viability was expressed as absorption at $490 \mathrm{~nm}$. The results shown are the mean of three independent experiments. Proliferation of BxPC-3 or Panc-1 cells infected with adenovirus vectors was slightly increased but there were no significant differences. p $>0.05$, bars, Standard error. (C) Colony formation assay was performed on BxPC-3 or Panc-1 cells infected with adenovirus for $48 \mathrm{~h}$. The number of colonies per well was counted 2 weeks after plating $1 \times 10^{3}$ cells. The cells without any infection were used as control. The data are the average of 3 independent triplicate experiments. PN, periostin.
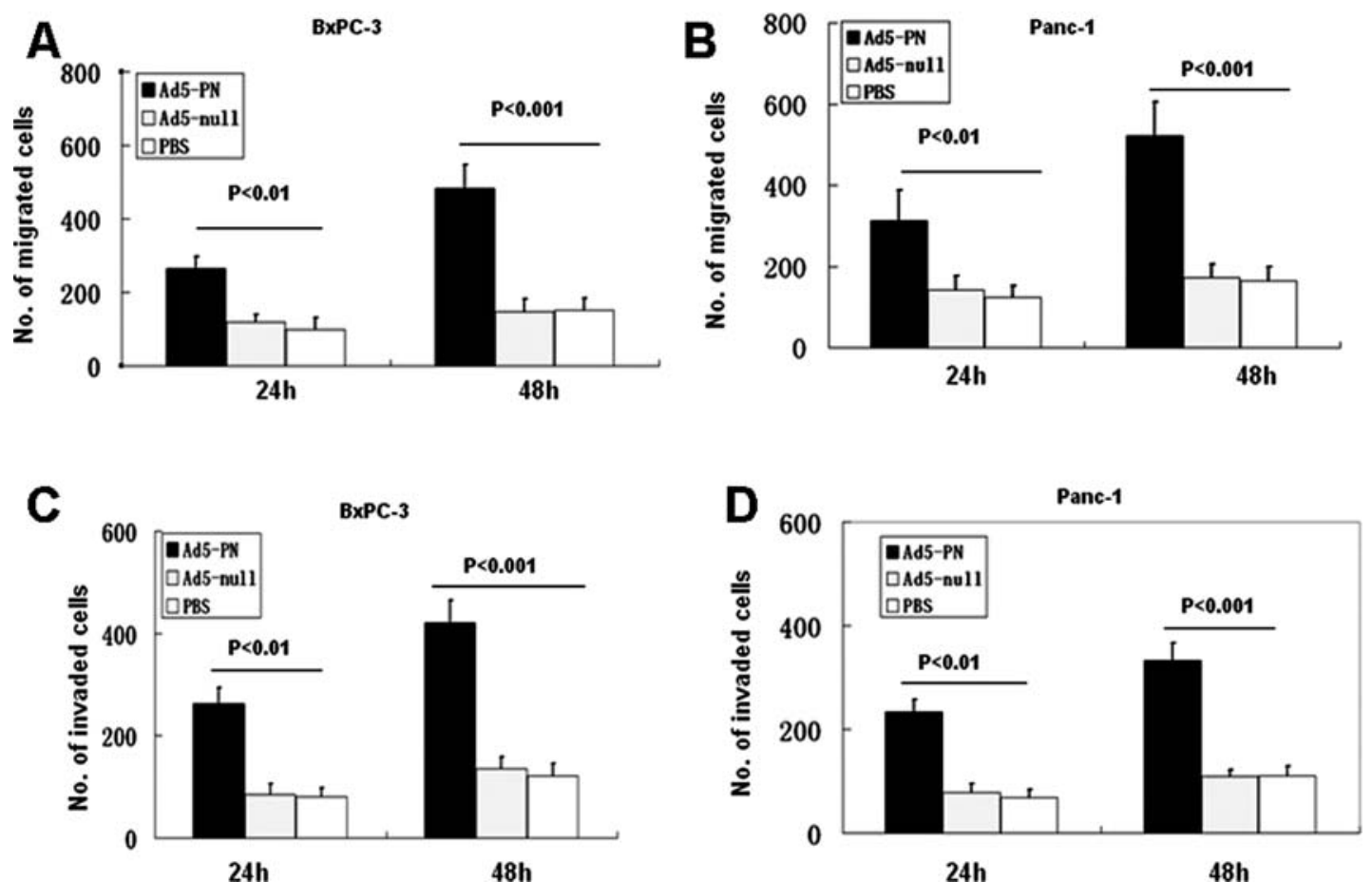

Figure 5. Effect of periostin on cell migration and invasion. Migration and invasion assays of BxPC-3 (A and C) or Panc-1 (B and D) cells infected with Ad5-PN . Statistically significant reductions were calculated by Student's t-test. (Top) In vitro motility potential of cells across transwell chambers was measured for BxPC-3 (A) or Panc-1 (B) cells. (Bottom) Cell invasion assays were done for BxPC-3 (C) or Panc-1 (D) cells by using matrigel coated transwell chambers. Invaded cells were counted in 10 random fields. Bovine serum albumin was used as a control. PN, periostin. 
tumors were strongly positive for periostin staining in the stroma, whereas about $30 \%$ showing strong periostin staining in neoplastic epithelium. These findings were in accordance with previous reports by Erkan et al (12), who identified periostin as a protein exclusively secreted by PSCs in pancreatic carcinoma. Building on these results and previous studies, we speculate that pancreatic cancer cell-mediated humoral factor(s) may stimulate periostin secreted from PSCs.

Overexpression of periostin has been reported to correlate with the progression of a number of human tumors, such as papillary thyroid carcinoma (30), NSCLC (28), breast cancer $(11,31)$ and melanoma $(32)$. In the present study, we found that the positive expression of periostin in the neoplastic stroma was clearly and significantly correlated with the degree of malignancy and poor prognosis. This observation is consistent with previous studies, which reported that elevated expression of periostin was associated with the invasion and progression of NSCLC, and periostin status acted as an prognostic factor in NSCLC $(28,33)$.

Accumulating evidence suggests that tumor microenvironment may produce many of the signals driving the proliferation and invasion of cancer cells $(34,35)$. The interactions between cancer and stromal cells, as well as between cancer cells and the extracellular matrix (ECM), are required in invasion and metastatic process. One critical step in tumor metastasis is termed epithelial-mesenchymal transition (EMT), which enables epithelial cancer cells to acquire invasive and metastatic potential $(36,37)$. Periostin has been shown to facilitate the migration and differentiation of cells that have undergone EMT, both during embryogenesis and in pathological conditions (38). Stable expression of periostin induces $293 \mathrm{~T}$ cells to undergo EMT and promotes cell migration, invasion, and adhesion (39). This is also true for NSCLC (28). Therefore, we hypothesized that a cancer microenvironment in which periostin is present may have a tumorsupportive phenotype.

The roles of periostin in tumorigenesis and development have been proposed previously. First, it is recognized that periostin has adhesive activity because its receptors, integrins, all mediate cell adhesion $(15,17,18)$ and the periostin protein shares structural and sequence homology with fasciclin I, which is an insect adhesion molecule $(4,18,40)$. Second, periostin promotes the migration, inhibits apoptosis and stimulates survival and growth of diverse cells $(9,15,18)$. Third, several studies have suggested that periostin increases tumor invasiveness by promoting tumor angiogenesis through complex signaling pathways, such as PI3kiase/Akt or focal adhesion kinase (FAK)-mediated signaling pathway $(15,29,41)$. In the present study, we established periostin ectopic expression pancreatic cancer cell lines and found that ectopic expression of periostin did not promote proliferation, but instead promoted anchorage-independent growth of pancreatic cancer cell lines. In addition, periostin significantly increased the migration and invasion of pancreatic cancer cells. Therefore, this study suggests that overexpression of periostin may confer on pancreatic cancer cells the ability to survive in the absence of anchorage and invade aggressively. However, the underlying mechanisms of invasion and anchorage-independent growth by periostin are still required.
Taken together, our studies have revealed a critical role of periostin in invasion and anchorage-independent growth in the metastatic process of pancreatic cancer. Importantly, periostin not only is associated with advanced pancreatic cancer but also is a prognostic marker for this disease. The findings that effect on metastatic potential of periostin raise the possibility that it could be used as a molecular target in the anti-metastatic therapy of pancreatic cancer.

\section{References}

1. Jemal A, Tiwari RC, Murray T, et al: Cancer statistics, 2004. CA Cancer J Clin 54: 8-29, 2004.

2. Guo $X$ and Cui Z: Current diagnosis and treatment of pancreatic cancer in China. Pancreas 31: 13-22, 2005

3. Okami J, Nakamori S, Hiraoka N, et al: Suppression of pancreatic cancer cell invasion by a cyclooxygenase-2-specific inhibitor. Clin Exp Metastasis 20: 577-584, 2003.

4. Takeshita S, Kikuno R, Tezuka K and Amann E: Osteoblastspecific factor 2: cloning of a putative bone adhesion protein with homology with the insect protein fasciclin I. Biochem J 294 (Pt 1): 271-278, 1993.

5. Horiuchi K, Amizuka N, Takeshita S, et al: Identification and characterization of a novel protein, periostin, with restricted expression to periosteum and periodontal ligament and increased expression by transforming growth factor beta. J Bone Miner Res 14: 1239-1249, 1999.

6. Litvin J, Zhu S, Norris R and Markwald R: Periostin family of proteins: therapeutic targets for heart disease. Anat Rec A Discov Mol Cell Evol Biol 287: 1205-1212, 2005.

7. Rios H, Koushik SV, Wang H, et al: Periostin null mice exhibit dwarfism, incisor enamel defects, and an early-onset periodontal disease-like phenotype. Mol Cell Biol 25: 11131-11144, 2005.

8. Shimazaki M, Nakamura K, Kii I, et al: Periostin is essential for cardiac healing after acute myocardial infarction. J Exp Med 205: 295-303, 2008.

9. Kudo Y, Ogawa I, Kitajima S, et al: Periostin promotes invasion and anchorage-independent growth in the metastatic process of head and neck cancer. Cancer Res 66: 6928-6935, 2006.

10. Grigoriadis A, Mackay A, Reis-Filho JS, et al: Establishment of the epithelial-specific transcriptome of normal and malignant human breast cells based on MPSS and array expression data. Breast Cancer Res 8: R56, 2006.

11. Puglisi F, Puppin C, Pegolo E, et al: Expression of periostin in human breast cancer. J Clin Pathol 61: 494-498, 2008.

12. Erkan M, Kleeff J, Gorbachevski A, et al: Periostin creates a tumor-supportive microenvironment in the pancreas by sustaining fibrogenic stellate cell activity. Gastroenterology 132: 1447-1464, 2007.

13. Streit S, Michalski CW, Erkan M, Friess H and Kleeff J: Confirmation of DNA microarray-derived differentially expressed genes in pancreatic cancer using quantitative RTPCR. Pancreatology 9: 577-582, 2009.

14. Ouyang G, Liu M, Ruan K, Song G, Mao Y and Bao S: Upregulated expression of periostin by hypoxia in non-smallcell lung cancer cells promotes cell survival via the Akt/PKB pathway. Cancer Lett 281: 213-219, 2009.

15. Bao S, Ouyang G, Bai X, et al: Periostin potently promotes metastatic growth of colon cancer by augmenting cell survival via the Akt/PKB pathway. Cancer Cell 5: 329-339, 2004.

16. Kim CJ, Yoshioka N, Tambe Y, Kushima R, Okada Y and Inoue H: Periostin is down-regulated in high grade human bladder cancers and suppresses in vitro cell invasiveness and in vivo metastasis of cancer cells. Int J Cancer 117: 51-58, 2005.

17. Baril P, Gangeswaran R, Mahon PC, et al: Periostin promotes invasiveness and resistance of pancreatic cancer cells to hypoxia-induced cell death: role of the beta4 integrin and the PI3k pathway. Oncogene 26: 2082-2094, 2007.

18. Gillan L, Matei D, Fishman DA, Gerbin CS, Karlan BY and Chang DD: Periostin secreted by epithelial ovarian carcinoma is a ligand for alpha(V)beta(3) and alpha(V)beta(5) integrins and promotes cell motility. Cancer Res 62: 5358-5364, 2002.

19. Ben QW, Wang JC, Liu J, et al: Positive expression of L1-CAM is associated with perineural invasion and poor outcome in pancreatic ductal adenocarcinoma. Ann Surg Oncol 17: 2213-2221, 2010. 
20. Sobin LH: TNM, sixth edition: new developments in general concepts and rules. Semin Surg Oncol 21: 19-22, 2003.

21. Ben QW, Zhao Z, Ge SF, Zhou J, Yuan F and Yuan YZ: Circulating levels of periostin may help identify patients with more aggressive colorectal cancer. Int J Oncol 34: 821-828, 2009.

22. Hezel AF, Kimmelman AC, Stanger BZ, Bardeesy N and Depinho RA: Genetics and biology of pancreatic ductal adenocarcinoma. Genes Dev 20: 1218-1249, 2006.

23. Jimeno A and Hidalgo M: Molecular biomarkers: their increasing role in the diagnosis, characterization, and therapy guidance in pancreatic cancer. Mol Cancer Ther 5: 787-796, 2006.

24. Mimeault M, Brand RE, Sasson AA and Batra SK: Recent advances on the molecular mechanisms involved in pancreatic cancer progression and therapies. Pancreas 31: 301-316, 2005.

25. Fukushima N, Kikuchi Y, Nishiyama T, Kudo A and Fukayama M: Periostin deposition in the stroma of invasive and intraductal neoplasms of the pancreas. Mod Pathol 21: 1044-1053, 2008.

26. Kanno A, Satoh K, Masamune A, et al: Periostin, secreted from stromal cells, has biphasic effect on cell migration and correlates with the epithelial to mesenchymal transition of human pancreatic cancer cells. Int J Cancer 122: 2707-2718, 2008

27. Kikuchi Y, Kashima TG, Nishiyama T, et al: Periostin is expressed in pericryptal fibroblasts and cancer-associated fibroblasts in the colon. J Histochem Cytochem 56: 753-764, 2008.

28. Soltermann A, Tischler V, Arbogast S, et al: Prognostic significance of epithelial-mesenchymal and mesenchymalepithelial transition protein expression in non-small cell lung cancer. Clin Cancer Res 14: 7430-7437, 2008.

29. Shao R, Bao S, Bai X, et al: Acquired expression of periostin by human breast cancers promotes tumor angiogenesis through up-regulation of vascular endothelial growth factor receptor 2 expression. Mol Cell Biol 24: 3992-4003, 2004.

30. Puppin C, Fabbro D, Dima M, et al: High periostin expression correlates with aggressiveness in papillary thyroid carcinomas. J Endocrinol 197: 401-408, 2008.
31. Quaresima B, Romeo F, Faniello MC, et al: BRCA1 5083del19 mutant allele selectively up-regulates periostin expression in vitro and in vivo. Clin Cancer Res 14: 6797-6803, 2008.

32. Tilman G, Mattiussi M, Brasseur F, van Baren N and Decottignies A: Human periostin gene expression in normal tissues, tumors and melanoma: evidences for periostin production by both stromal and melanoma cells. Mol Cancer 6: 80, 2007.

33. Takanami I, Abiko T and Koizumi S: Expression of periostin in patients with non-small cell lung cancer: correlation with angiogenesis and lymphangiogenesis. Int J Biol Markers 23: 182-186, 2008.

34. Hanahan D and Weinberg RA: The hallmarks of cancer. Cell 100: 57-70, 2000.

35. Bissell MJ and Radisky D: Putting tumours in context. Nat Rev Cancer 1: 46-54, 2001.

36. Peinado H, Olmeda D and Cano A: Snail, Zeb and bHLH factors in tumour progression: an alliance against the epithelial phenotype? Nat Rev Cancer 7: 415-428, 2007.

37. Thiery JP and Sleeman JP: Complex networks orchestrate epithelial-mesenchymal transitions. Nat Rev Mol Cell Biol 7: 131-142, 2006.

38. Lindsley A, Snider P, Zhou H, et al: Identification and characterization of a novel Schwann and outflow tract endocardial cushion lineage-restricted periostin enhancer. Dev Biol 307: 340-355, 2007.

39. Yan W and Shao R: Transduction of a mesenchyme-specific gene periostin into $293 \mathrm{~T}$ cells induces cell invasive activity through epithelial-mesenchymal transformation. J Biol Chem 281: 19700-19708, 2006.

40. Sasaki H, Sato Y, Kondo S, et al: Expression of the periostin mRNA level in neuroblastoma. J Pediatr Surg 37: 1293-1297, 2002.

41. Siriwardena BS, Kudo Y, Ogawa I, et al: Periostin is frequently overexpressed and enhances invasion and angiogenesis in oral cancer. Br J Cancer 95: 1396-1403, 2006. 